\title{
Modelling of non-steady-state concentration profiles at ISFET-based coulometric sensor-actuator systems
}

\author{
W. OLTHUIS *, J LUO, B H VAN DER SCHOOT and P BERGVELD \\ Department of Electrical Engineering, Unversity of Twente, P.O. Box 217, 7500 AE Enschede (The Netherlands) \\ M BOS and W E VAN DER LINDEN \\ Department of Chemical Technology, Unversity of Twente, P.O. Box 217, 7500 AE Enschede (The Netherlands)
}

(Received 30th May 1989)

SUMMARY

Acid or base concentrations can be determined very rapidly by performing an acid-base titration with coulometncally generated $\mathrm{OH}^{-}$or $\mathrm{H}^{+}$ions at a noble metal actuator electrode in close proximity to the pH-sensitive gate of an ion-sensitive field effect transistor (ISFET) The ISFET is used as the indicator electrode to detect the equivalence point in the titration curve Typical values for the time needed to reach the equivalence point are $0.5-10 \mathrm{~s}$ for acid or base concentrations ranging from $05 \times 10^{-3}$ to $20 \times 10^{-3}$ $\mathrm{mol} \mathrm{1}^{-1}$

A model is presented, giving an analytical description of the diffusion phenomena that occur with the sensor-actuator system. The results of this analytical model description, using linearized concentration gradients, are presented together with the results of numerical simulations Both results are in good agreement with measurements.

Coulometric generation of $\mathrm{H}^{+}$or $\mathrm{OH}^{-}$ions at an actuator electrode permits the control of the chemical environment at the surface of that electrode. By placing a chemical sensor, e.g., a pHsensitive ion-sensitive field effect transistor (ISFET), near the actuator electrode, one can measure the resulting concentration change of $\mathrm{H}^{+}$ or $\mathrm{OH}^{-}$ions. The rate of this change is related to the bulk concentrations of the buffering components in the solution. Hence, in addition to the flow of data from the chemical sensing device to the measuring system in conventional chemical sensors, there is also information flowing from the controller to the actuator electrode, which allows the control of the chemical environment locally.

$\mathrm{H}^{+}$or $\mathrm{OH}^{-}$can be generated at a noble metal actuator electrode by the electrolysis of water.
Depending on the direction of the actuator current, one of the following reactions occurs:

$$
\begin{aligned}
& 2 \mathrm{H}_{2} \mathrm{O} \rightarrow 4 \mathrm{H}^{+}+4 \mathrm{e}^{-}+\mathrm{O}_{2} \\
& 2 \mathrm{H}_{2} \mathrm{O}+2 \mathrm{e}^{-} \rightarrow 2 \mathrm{OH}^{-}+\mathrm{H}_{2}
\end{aligned}
$$

The resulting local decrease or increase in the $\mathrm{pH}$ can be measured by an integrated $\mathrm{pH}$-sensitive ISFET.

The coulometric sensor-actuator system is shown schematically in Fig. 1. The chip is mounted on a carrier and is used as a dipstick to measure in samples of typically 10-20 ml. The change in $\mathrm{pH}$ only takes place in a small volume formed by the area of the actuator electrode, typically $1 \mathrm{~mm}^{2}$, and the thickness of the diffusion layer. Hence the measurement is carried out in a volume of a few microlitres in the proximity of the sensor-actuator 


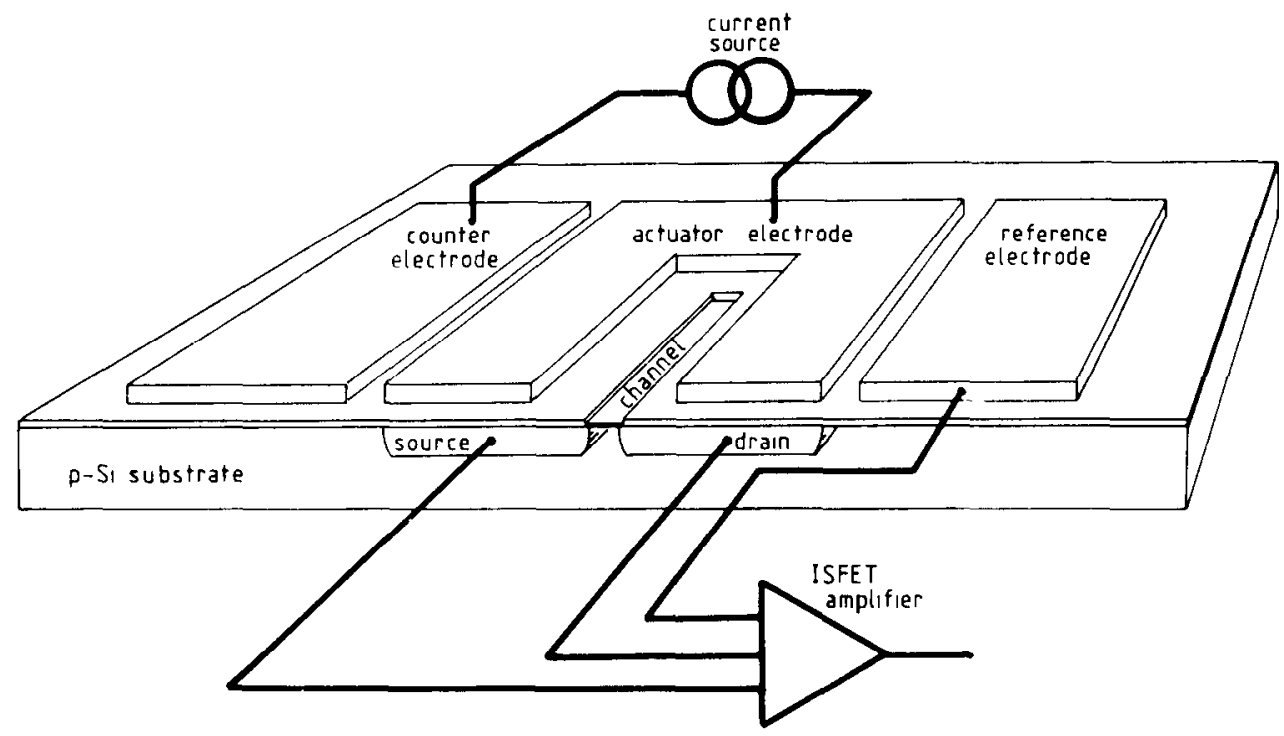

Fig 1. Basic components of the acid-base concentration sensor.

system, while the remainder of the sample solution is unaffected.

The application of the sensor-actuator system that will be dealt with in this paper is that of an acid-base concentration sensor. By considering the generated ions as the titrant, it is possible to effect a coulometric titration. The time $t_{\text {eq }}$ necessary to reach the equivalence point in the titration curve measured by the ISFET sensor is related to the acid or base concentration of the bulk solution via the process of free diffusion [1].

The sensor-actuator system is placed in an unstirred aqueous solution of acid HA. At $t=0 \mathrm{a}$ cathodic current pulse is applied to the actuator electrode, causing the generation of $\mathrm{OH}^{-}$ions according to Eqn. 2. Because the dissociation of the acid is assumed to be fast and proton-hydroxyl equilibrium of water is very rapidly established, HA molecules near the actuator electrode surface are depleted, causing a decreasing concentration of HA from the bulk of the solution towards the actuator electrode surface.

During this process, the $\mathrm{pH}$ at the sensor surface is continuously measured and thus the equivalence point in the titration curve can be determined. Because the ISFET is only used as a (fast) dynamic indicator with a typical response time of the order of milliseconds [2], this method of measuring is not influenced by long-term drift of the ISFET. The time $t_{\text {eq }}$ needed to reach the equivalence point at the sensor surface is dependent on the bulk concentration of the acid in addition to the actuator current density, and is described by the laws of mass transfer. The nonzero distance between the sensor and actuator, both positioned within the microlitre volume in which the titration takes place, is a complicating factor.

Two other applications of the sensor-actuator system are described. The first is a carbon dioxide sensor. On top of the device as depicted in Fig. 1, a thin electrolyte layer can be applied separated from the environment by a $\mathrm{CO}_{2}$-permeable membrane. Thus a coulometric carbon dioxide sensor is created in which the amount of $\mathrm{CO}_{2}$ in the internal electrolyte, which is equilibrated with the environment, is determined by means of coulometric titration [3]. The slow hydration reaction of $\mathrm{CO}_{2}$ prevents a direct titration. Therefore, first an excess of $\mathrm{OH}^{-}$ions has to be generated. At the resulting high $\mathrm{pH}$, the conversion of $\mathrm{CO}_{2}$ to $\mathrm{HCO}_{3}^{-}$ ions occurs relatively rapidly and after a few seconds the excess of hydroxyl ions can be determined in a back-tıtration with protons gener- 
ated by coulometry. From the result of this titration, the original $\mathrm{CO}_{2}$ concentration can be calculated.

The second application is an enzyme sensor. The integration of a membrane with immobilized enzymes on an ISFET with a noble metal actuator electrode as shown in Fig. 1 offers the possibility of controlling the $\mathrm{pH}$ inside the membrane. By using a feedback system, the products of the enzymatic reaction in the membrane are continuously titrated [4]. Hence the sensor becomes independent of the buffer capacity of the sample solution and the required actuator current depends linearly on the substrate concentration. Because the enzyme is operating at a constant $\mathrm{pH}$, the linear response range is also greatly expanded.

This paper focuses on the coulometric acid-base concentration sensor; however, the theoretical description of this basic device can also be applied to the other examples mentioned. Mass transfer is described in terms of free diffusion and the theoretical results are compared with corresponding measurements. Apart from an analytical model description, a numerical solution of the equations describing diffusion is presented. The results of the numerical solution are used to check readily the measurements and to obtain an indication of the form of the concentration profiles at the actuator electrode.

\section{MODELLING OF MASS TRANSFER}

The general mass transfer equation is composed of three flux terms:

$J_{\text {tot }, l}=J_{\text {diffusion }, \imath}+J_{\text {migration }, l}+J_{\text {convection }, l}$

where $J_{\text {tot }, l}\left(\mathrm{~mol} \mathrm{~s}^{-1} \mathrm{~m}^{-2}\right)$ is the total flux of the species $i$. Convective transfer is considered to be absent, because significant (thermal) density gradients in the non-stirred solution can be avoided.

In general, migration can be avorded by a sufficiently large excess of supporting electrolyte concentration. One must bear in mind, however, that the concentration of the generated ions near the actuator electrode could be comparable to the supporting electrolyte concentration, possibly causing a migration flux that is no longer negligible. This case will be considered in a later paper and will be neglected here.

Further, only sem1-infinite diffusive mass transfer in one dimension is taken into account; the noble metal actuator electrode of the sensor-actuator device used in the experiments is considered to be a planar electrode.

\section{Analytıcal description: rigorous approach}

Consider an acidic solution. For $t=0$, $C_{\mathrm{HA}}(x, t) \gg C_{\mathrm{OH}}(x, t)$, where $C_{l}(x, t)\left(\mathrm{mol} \mathrm{m}^{-3}\right)$ represents the concentration of species $t$, so that the diffusion equation, using Fick's second law of diffusion, becomes

$\partial C_{\mathrm{HA}}(x, t) / \partial t=D_{\mathrm{HA}} \partial^{2} C_{\mathrm{HA}}(x, t) / \partial x^{2}$

where $D_{\mathrm{HA}}\left(\mathrm{m}^{2} \mathrm{~s}^{-1}\right)$ is the diffusion coefficient of acid HA, with initial and boundary conditions

$\left.\begin{array}{l}t=0, \text { all } x \\ x \rightarrow \infty, \text { all } t\end{array}\right\} \rightarrow\left\{\begin{array}{l}C_{\mathrm{HA}}(x, t)=C_{\mathrm{HA}, \text { bulk }} \\ C_{\mathrm{OH}}(x, t) \rightarrow 0\end{array}\right.$

At $t=0$ the cathodic actuator current is switched on, resulting in a constant current density $J_{c}\left(\mathrm{~A} \mathrm{~m}^{-2}\right)$ at the actuator electrode where $\mathrm{OH}^{-}$ions are now generated according to Eqn. 2. The dissociation of acid HA and the establishment of the water equilibrium are considered to be instantaneous, so that, by the use of Fick's first law of diffusion

$D_{\mathrm{HA}} \partial C_{\mathrm{HA}}(x, t) /\left.\partial x\right|_{x=0}=J_{\mathrm{c}} / F$

where $F\left(\mathrm{C} \mathrm{mol}^{-1}\right)$ is the Faraday constant.

Using Laplace transformation to solve Eqns. $4-6$ results in [5]

$$
\begin{aligned}
C_{\mathrm{HA}}(x, t) \\
=C_{\mathrm{HA}, \text { bulk }}-\frac{J_{\mathrm{c}}}{F D_{\mathrm{HA}}}\left[2\left(\frac{D_{\mathrm{HA}} t}{\pi}\right)^{1 / 2} \exp \left(\frac{-x^{2}}{4 D_{\mathrm{HA}} t}\right)\right. \\
\left.-x \operatorname{erfc}\left(\frac{x}{2\left(D_{\mathrm{HA}} t\right)^{1 / 2}}\right)\right]
\end{aligned}
$$

Some concentration profiles $C_{\mathrm{HA}}(x, t)$ at various times during the constant actuator current pulse are given in Fig. 2. 


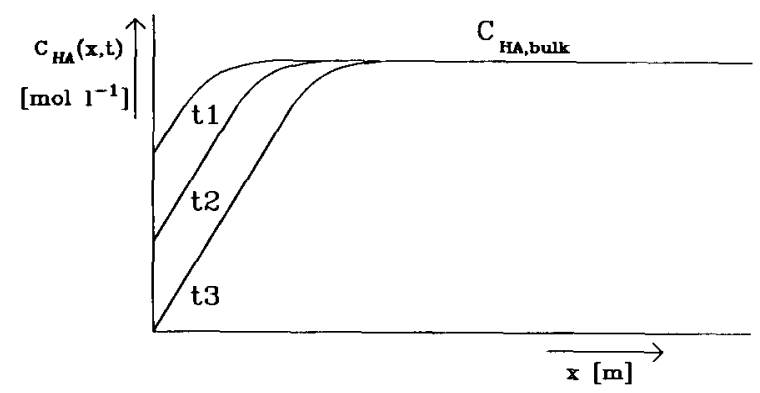

Fig 2 Concentration profiles at the actuator electrode during a constant actuator current pulse at three consecutive times

At $t=t_{3}$ in Fig. 2, the HA concentration at the actuator interface at $x=0$ just reaches zero. This time, at which $C_{\mathrm{HA}}(0, t)$ drops to zero, is called ${ }^{t}$ Sand and is defined by substituting $x=0$ into Eqn. 7, which leads to the well-known Sand equation [5]:

$$
\begin{aligned}
& C_{\mathrm{HA}}\left(0, t_{\text {Sand }}\right) \\
& \quad=0=C_{\text {HA,bulk }}-\left(2 J_{\mathrm{c}} / F\right)\left(t_{\text {Sand }} / \pi D_{\mathrm{HA}}\right)^{1 / 2}
\end{aligned}
$$

or

$$
t_{\text {Sand }}=\left[\left(C_{\mathrm{HA}, \text { bulk }} F\left(\pi D_{\mathrm{HA}}\right)^{1 / 2}\right) / 2 j_{\mathrm{c}}\right]^{2}
$$

The time $t_{\mathrm{eq}}$ that is needed to reach the equivalence point would be equal to $t_{\text {sand }}$ if the position of the sensor coincided exactly with the position of the actuator electrode at $x=0$. In practice, this is technologically impossible and therefore the sensor is considered to be placed at $x_{\mathrm{s}}$ with respect to the actuator electrode which is located at $x=0$ (one-dimensional case to make the description of the model not unnecessarily complex). For $t>t_{\text {Sand }}$, the boundary condition $C_{\mathrm{HA}}(x, t) \gg$ $C_{\mathrm{OH}}(x, t)$ is no longer valid for $x=0$ and Eqn. 7 can no longer be used. Extra boundary conditions must be added to the existing model to cope with the concentration gradient of $C_{\mathrm{OH}}(x, t)$ where $C_{\mathrm{OH}}(x, t)>C_{\mathrm{HA}}(x, t)$, caused by the continuous generation of $\mathrm{OH}^{-}$ions at the actuator electrode for $t>t_{\text {Sand }}$.

The concentration gradient of $C_{\mathrm{OH}}(x, t)$ gives rise to diffusion from the actuator electrode surface into the acidic bulk solution. The reaction plane $\lambda(t)$ is the plane where the two concentration profiles of $C_{\mathrm{OH}}(x, t)$ and $C_{\mathrm{HA}}(x, t)$ meet as indicated in Fig. 3.

At $\lambda(t)$ both $C_{\mathrm{OH}}(x, t)$ and $C_{\mathrm{HA}}(x, t)$ are considered to be zero, representing the plane where the equivalence point in the coulometric acid-base titration is reached. Hydroxyl cons are continuously generated as long as the actuator current is switched on. This causes the reaction plane $\lambda(t)$ to move into the bulk solution with increasing time $t$.

As can be deduced from Fig. 3, the time $t_{\text {eq }}$ that is needed to reach the equivalence point at the position of the sensor $x_{\mathrm{s}}$ is the sum of two terms: the time $t_{\text {Sand }}$ needed to let $C_{\mathrm{HA}}(0, t)$ drop to zero, according to Eqn. 9, and the time $t_{\text {delay }}$ needed for the reaction plane $\lambda(t)$ to reach $x_{\mathrm{s}}$.

The necessary equations describing the model for $t \geqslant t_{\text {Sand }}$ are the differential equations describing diffusion:

$$
\begin{aligned}
& \partial C_{\mathrm{OH}}(x, t) / \partial t=D_{\mathrm{OH}} \partial^{2} C_{\mathrm{OH}}(x, t) / \partial x^{2} \\
& x<\lambda(t) \\
& \partial C_{\mathrm{HA}}(x, t) / \partial t=D_{\mathrm{HA}} \partial^{2} C_{\mathrm{HA}}(x, t) / \partial x^{2} \\
& \quad x>\lambda(t)
\end{aligned}
$$

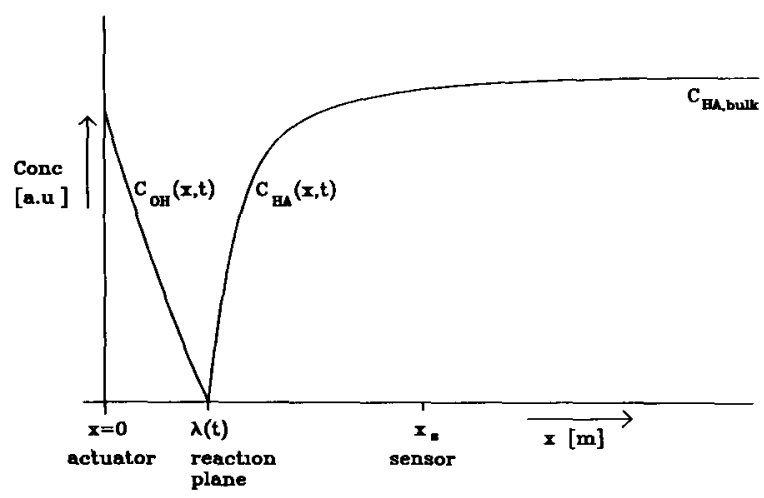

Fig. 3. Concentration profiles at the actuator electrode obtained from numerical solution and expressed in arbitrary units for $t>t_{\text {Sand }}$ or $\lambda(t)>0$ For $x<\lambda(t)$ the $\mathrm{OH}^{-}$concentration profile is drawn and for $x>\lambda(t)$ the HA concentration profile is drawn. The two profules meet at the reaction plane $\lambda(t)$. 
The initial and boundary conditions are

$$
\begin{aligned}
& t=t_{\text {Sand }}: C_{\mathrm{HA}}(x, t)=C_{\mathrm{HA}}\left(x, t_{\text {Sand }}\right) \\
& \left.\begin{array}{l}
x=\lambda(t): C_{\mathrm{HA}}(x, t)=C_{\mathrm{OH}}(x, t) \rightarrow 0 \\
x=0: D_{\mathrm{OH}} \partial C_{\mathrm{OH}}(x, t) / \partial x=-\jmath_{\mathrm{c}} / F
\end{array}\right\}
\end{aligned}
$$

An extra boundary condition is obtained from the fact that each mole of hydroxyl ions "consumes" an equal amount of acid at the reaction plane $\lambda(t)$. This results in the flux balance at $x=\lambda(t)$

$$
\begin{aligned}
& D_{\mathrm{HA}} \partial C_{\mathrm{HA}}(x, t) / \partial x \\
& \quad=-D_{\mathrm{OH}} \partial C_{\mathrm{OH}}(x, t) /\left.\partial x\right|_{x=\lambda(t)}
\end{aligned}
$$

Rigorous analytical solutions to the equations which describe the moving reaction plane model with simpler initial and boundary conditions than stated here are known [6,7] and are very elaborate [constant concentrations of both the diffusing species at $x<\lambda(t)$ and at $x>\lambda(t)]$. However, a rigorous analytical solution to the problem given here, having one current-controlled continuous source for the species at $x<\lambda(t)$ and one constant concentration source for the species at $x>$ $\lambda(t)$, is very complicated. To obtain an analytical solution, the model is simplified by linearizing the concentration gradients.

Analytical description: linearized concentration gradients for $\mathrm{t}>\mathrm{t}_{\text {Sand }}$

The description of the model using linearized concentration gradients starts at $t=t_{\text {Sand }}$. The thickness of the diffusion layer $\delta_{\text {Sand }}$, which is needed for the calculations in this section, is obtained by the condition that the number of depleted HA molecules due to the generated $\mathrm{OH}^{-}$ ions at the actuator electrode for the linearized gradient (Fig. 4, curve 2) equals the number of depleted HA molecules for the gradient obtained by the rigorous analytical solution (Eqn. 7) as lllustrated in Fig. 4, curve 1.

From Fig. 4, the expression for the total number of depleted HA molecules $Q_{\text {depl,Sand }}$ is obtained:

$$
Q_{\mathrm{depl}, \text { Sand }}=0.5 A \delta_{\mathrm{Sand}} C_{\mathrm{HA}, \mathrm{bulk}}=\left(J_{\mathrm{c}} A / F\right) t_{\text {Sand }}
$$

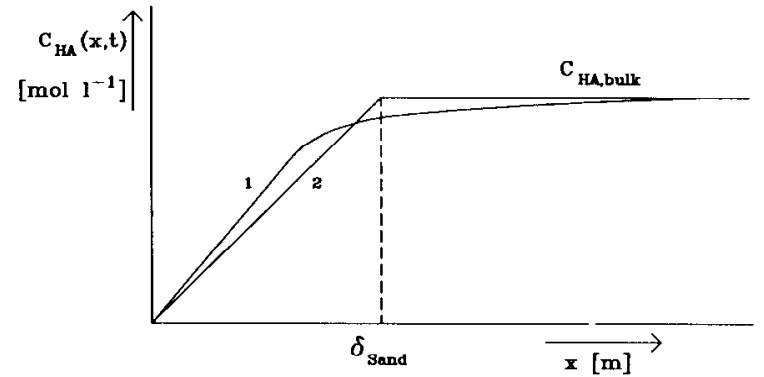

Fig. 4 The exact concentration profile of $C_{\mathrm{HA}}(x, t)$ (curve 1) and the linearized profile (curve 2) at $t=t_{\text {Sand }}$.

where $A\left(\mathrm{~m}^{2}\right)$ is the surface area of the actuator electrode. Substituting the equation for $t_{\text {Sand }}$ (Eqn. 9) into Eqn. 15 yields

$\delta_{\text {Sand }}=\pi C_{\mathrm{HA}, \text { bulk }} F D_{\mathrm{HA}} / 2 J_{\mathrm{c}}$

Then, for $t>t_{\text {Sand }}$ or $\lambda(t)>0$, according to the linearized model description, Fig. 3 can now be replaced by Fig. 5 .

A consequence of linearizing the concentration gradients is the constant flux $J_{\mathrm{HA}}\left(\mathrm{mol} \mathrm{m}^{-2} \mathrm{~s}^{-1}\right)$ in the film $\lambda(t)<x<\lambda(t)+\delta_{\mathrm{HA}}$, resulting in a parallel moving HA concentration profile for $t>$ $t_{\text {Sand }}$, or:

$\delta_{\mathrm{HA}}=\delta_{\text {Sand }} \quad$ for all $t \geqslant t_{\text {Sand }}$

From Fig. 5 and Eqn. 17, the flux $J_{\mathrm{HA}}$ in the film $\lambda(t)<x<\lambda(t)+\delta_{\mathrm{HA}}$ can be obtained:

$$
\begin{aligned}
J_{\mathrm{HA}} & =-D_{\mathrm{HA}} \partial C_{\mathrm{HA}}(x, t) /\left.\partial x\right|_{\lambda(t)<x<\lambda(t)+\delta_{\mathrm{HA}}} \\
& =-D_{\mathrm{HA}} C_{\mathrm{HA}, \text { bulk }} / \delta_{\mathrm{HA}}=-2 J_{\mathrm{c}} / \pi F
\end{aligned}
$$

The net flux $J_{\text {net }}$ in the film $0<x<\lambda(t)$ can be obtained by subtracting the $\mathrm{OH}^{-}$ion flux at the

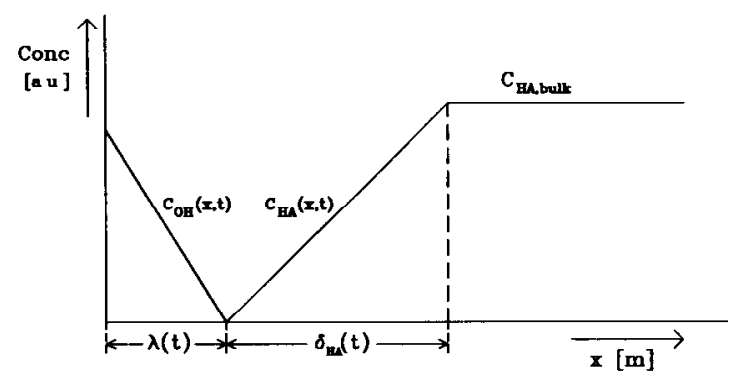

F1g. 5. The linearized concentration profiles for $t>t_{\text {Sand }}$; the profile for $\mathrm{OH}^{-}$ions for $x<\lambda(t)$ and for HA for $x>\lambda(t)$. The profiles intersect at the reaction plane $\lambda(t)$. 
reaction plane $\lambda(t)$ from the incoming $\mathrm{OH}^{-}$ion flux at $x=0$ by generation:

$J_{\text {net }}=J_{\mathrm{OH}, \text { in }}-J_{\mathrm{OH} \text {,out }}$

The absolute value of flux $J_{\mathrm{OH} \text {,out }}$ equals the flux $J_{\mathrm{HA}}$ to maintain the HA gradient as expressed by Eqn. 18, but is opposite in sign owing to the opposite direction of the flux:

$J_{\mathrm{OH}, \mathrm{out}}=-J_{\mathrm{HA}}$

The incoming flux by $\mathrm{OH}^{-}$generation is

$J_{\mathrm{OH}, \mathrm{n}}=J_{\mathrm{c}} / F$

Substitution of Eqns. 20 and 21 in Eqn. 19 results in

$$
\begin{aligned}
J_{\text {net }} & =(\pi-2) j_{\mathrm{c}} / \pi F \\
& =-D_{\mathrm{OH}} \partial C_{\mathrm{OH}}(x, t) /\left.\partial x\right|_{0<x<\lambda(t)} \\
& =D_{\mathrm{OH}} C_{\mathrm{OH}}(0, t) / \lambda(t)
\end{aligned}
$$

or

$\lambda(t)=\pi C_{\mathrm{OH}}(0, t) F D_{\mathrm{OH}} /(\pi-2) j_{\mathrm{c}}$

One extra condition is needed to solve Eqn. 23: $C_{\mathrm{OH}}(0, t)$ is still unknown. This extra condition is obtained by considering the effect of the total amount of charge $Q_{\text {tot }}$ injected by the actuator electrode in the solution to form $\mathrm{OH}^{-}$ions after $t_{\text {Sand }}$ :

$Q_{\mathrm{tot}}=J_{\mathrm{c}} A t / F$

where $A\left(\mathrm{~m}^{2}\right)$ is the surface area of the actuator electrode.

These generated $\mathrm{OH}^{-}$ions are partly consumed to deplete the HA concentration. From Fig. 6 and Eqn. 17, the amount of $\mathrm{OH}^{-}$ions used to deplete the HA molecules $Q_{\text {depl }}$ after $t=t_{\text {Sand }}$ is obtained: $Q_{\text {depl }}=A C_{\mathrm{HA}, \text { bulk }} \lambda(t)$

The remainder of the total amount of generated $\mathrm{OH}^{-}$ions after $t_{\text {Sand }}$ is still present as $\mathrm{OH}^{-}$ions. From Fig. 6 this amount $Q_{\mathrm{OH}}$ is obtained:

$Q_{\mathrm{OH}}=0.5 A C_{\mathrm{OH}}(0, t) \lambda(t)$

Considering that

$Q_{\mathrm{tot}}=Q_{\mathrm{depl}}+Q_{\mathrm{OH}}$

and substituting Eqns. 24-26 into Eqn. 27 results in

$\lambda(t)=2 J_{\mathrm{c}} t /\left\{F\left[C_{\mathrm{OH}}(0, t)+2 C_{\mathrm{HA}, \mathrm{bulk}}\right]\right\}$

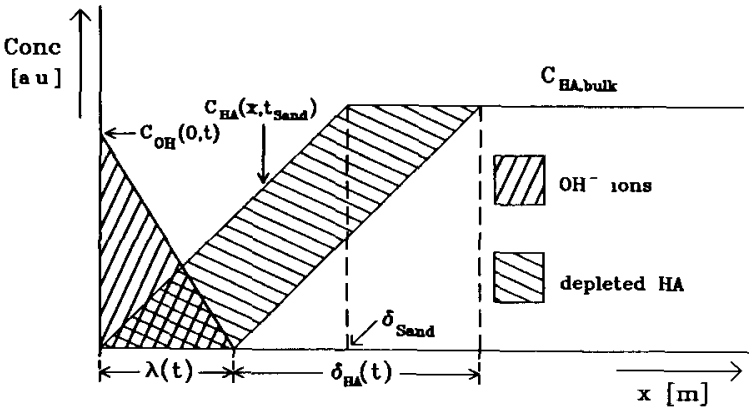

Fig 6. Effect of $\mathrm{OH}^{-}$ion generation for $t>t_{\text {Sand }}$; some of the tons are consumed by reaction with $\mathrm{HA}$, resulting in a local $\mathrm{HA}$ depletion, and some are present as $\mathrm{OH}^{-}$1ons, forming a concentration gradient.

Eqns. 23 and 28 form a set of two independent equations with $\lambda(t)$ and $C_{\mathrm{OH}}(0, t)$ as unknown variables. Solving this set results in

$t=\left[(\pi-2) \lambda^{2}(t) / 2 \pi D_{\mathrm{OH}}\right]+\left[F C_{\mathrm{HA}, \text { bulk }} \lambda(t) / J_{\mathrm{c}}\right]$

By substituting $\lambda(t)=x_{s}$, the position of the sensor, in Eqn. 29 the delay time $t_{\text {delay }}$ is obtained; $t_{\text {delay }}$ is the time it takes for the reaction plane to reach the position where the sensor is placed.

The time $t_{\mathrm{eq}}$ needed to reach the equivalence point in the titration curve is the sum of the result of Eqn. 9, being the time $t_{\text {Sand }}$ needed to deplete totally the acid concentration at $x=0$, and of Eqn. 29, where $t_{\text {delay }}$ is obtained for $\lambda(t)=x_{\mathrm{s}}$ :

$$
\begin{aligned}
t_{\text {eq }}= & t_{\text {Sand }}+t_{\text {delay }} \\
= & {\left[\frac{C_{\mathrm{HA}, \text { bulk }} F\left(\pi D_{\mathrm{HA}}\right)^{1 / 2}}{2 J_{\mathrm{c}}}\right]^{2}+\frac{(\pi-2) x_{\mathrm{s}}^{2}}{2 \pi D_{\mathrm{OH}}} } \\
& +\frac{F C_{\mathrm{HA}, \text { bulk }} x_{\mathrm{s}}}{J_{\mathrm{c}}}
\end{aligned}
$$

Eqn. 30 can be approximated for large $C_{\text {HA,bulk }}$ by

$$
t_{\mathrm{eq}}^{1 / 2}=\frac{F C_{\mathrm{HA}, \text { bulk }}\left(\pi D_{\mathrm{HA}}\right)^{1 / 2}}{2 J_{\mathrm{c}}}+\left(\frac{\pi-2}{2 \pi D_{\mathrm{OH}}}\right)^{1 / 2} \cdot x_{\mathrm{s}}
$$

\section{Numerical solution}

A numerical solution of the previously stated model does not give much insight into the way in which the model depends on the parameters de- 
scribing it. The numerical solution, however, can readily be used to compare the result with the analytical description and with the corresponding measurements.

To approach the model which describes the diffusion mechanism in a numerical way, Eqn. 4 can be written as a finite difference expression, a discretization in time and space [8], using

$$
\left.\begin{array}{c}
\mathrm{d} C_{l} / \mathrm{d} t=\left(C_{t}^{\prime}-C_{t}\right) / \Delta t \quad \Delta t=t^{\prime}-t \\
\mathrm{~d}^{2} C_{t} / \mathrm{d} x^{2}=\left(C_{t+1}-2 C_{t}+C_{t-1}\right) /(\Delta x)^{2} \\
\Delta x=x_{t+1}-x_{l}
\end{array}\right\}
$$

Substitution of Eqn. 32 in Eqn. 4 yields

$$
\begin{gathered}
C_{t}^{\prime}=C_{t}+m\left(C_{t+1}-2 C_{t}+C_{t-1}\right) \\
m=D \Delta t /(\Delta x)^{2}
\end{gathered}
$$

To guarantee stability, $m$ must be smaller than 0.5 . The controlled current boundary condition of Eqn. 6 is satisfied by

$$
C_{0}=C_{1}-\left(J_{\mathrm{c}} / F D\right)
$$

In the case of diffusion in a fully dissociated acid, only the diffusion of $\mathrm{H}^{+}$and $\mathrm{OH}^{-}$ions has to be considered. Equation 33 is used for both species. After each time-iteration step, equilibrium has to be re-established by

$$
\left.\begin{array}{l}
C_{\mathrm{H}, t, \mathrm{eq}} C_{\mathrm{OH}, t, \mathrm{eq}}=K_{\mathrm{W}} \\
\text { and } \\
C_{\mathrm{H}, t, \mathrm{eq}}-C_{\mathrm{OH}, t, \mathrm{eq}}=C_{\mathrm{H}, \imath}-C_{\mathrm{OH}, l}
\end{array}\right\}
$$

Solving Eqn. 35 for $C_{\mathrm{H}_{\text {, }, \text { eq }}}$ yields

$$
\begin{aligned}
C_{\mathrm{H}, \imath, \mathrm{eq}}= & 0.5\left\{C_{\mathrm{H}, \iota}-C_{\mathrm{OH}, t}\right. \\
& \left.+\left[\left(C_{\mathrm{H}, \iota}-C_{\mathrm{OH}, \iota}\right)^{2}+4 K_{\mathrm{W}}\right]^{1 / 2}\right\}
\end{aligned}
$$

In the case of diffusion in a partly dissociated acid, e.g., acetic acid (HAc), the diffusion of $\mathrm{H}^{+}$, $\mathrm{OH}^{-}$and $\mathrm{Ac}^{-}$ions in addition to HAc molecules has to be taken into account. Then, after each time-iteration step, equilibrium has to be calculated by the use of the following:

$$
\begin{aligned}
& \text { acid dissociation equilibrium: } \\
& C_{\mathrm{H}, t, \mathrm{eq}} C_{\mathrm{AC}, l, \mathrm{eq}}=K_{\mathrm{a}} C_{\mathrm{HAc}, t, \mathrm{eq}} \\
& \text { mass balance: } \\
& C_{\mathrm{HAc}, t, \text { eq }}+C_{\mathrm{AC}, t, \mathrm{eq}}=C_{\mathrm{HAc}, l}+C_{\mathrm{AC}, t} \\
& \text { water equilibrıum: } \\
& C_{\mathrm{H}, t, \mathrm{eq}} C_{\mathrm{OH}, t, \mathrm{eq}}=K_{\mathrm{W}} \\
& \text { neutrality: } \\
& \begin{array}{c}
C_{\mathrm{H}, l, \mathrm{eq}}-C_{\mathrm{OH}, l, \mathrm{eq}}-C_{\mathrm{AC}, l, \mathrm{eq}} \\
=C_{\mathrm{H}, t}-C_{\mathrm{OH}, t}-C_{\mathrm{AC}, t}
\end{array}
\end{aligned}
$$

The conditions stated in Eqn. 37 result, with respect to the proton equilibrium concentration $C_{\mathrm{H}, l, \text { eq }}$, in

$$
\begin{aligned}
& x^{3}+\left(K_{\mathrm{a}}+\beta\right) x^{2}-\left[K_{\mathrm{W}}+K_{\mathrm{a}}(\epsilon-\beta)\right] x-K_{\mathrm{W}} K_{\mathrm{a}} \\
& \quad=0
\end{aligned}
$$

where $x=C_{\mathrm{H}, t, \mathrm{eq}}, \beta=-C_{\mathrm{H}, t}+C_{\mathrm{OH}, t}+C_{\mathrm{AC}, t}$ and $\epsilon=C_{\mathrm{HAc}, l}+C_{\mathrm{Ac}, l}$. This cubic equation can be solved by the use of Cardan's rule.

\section{EXPERIMENTAL}

Sensor-actuator device

As mentioned in the introduction, the pH-sensitive ISFET is used as the sensing device. The ISFET is fabricated following the standard NMOS processing steps [9], with an added tantalum oxide gate dielectric on top of the silicon oxide. For the experiments mentioned here, two types of actuator electrodes are used. The first type is deposited around the gate and consists of a thin gold film $(0.6 \mu \mathrm{m})$ evaporated on top of a $0.1-\mu \mathrm{m}$ silver film and a $20-\mathrm{nm}$ titanium film for protection of the ISFET against mobile gold atoms and good adhesion of the film on the underlaying tantalum oxide, respectively. Using standard photolithographic techniques, the actuator electrode is shaped as shown in Fig. 7ab. The active area of the actuator electrode is $1 \mathrm{~mm}^{2}$ and is determined by a layer of polyimide, which protects the remainder of the device from contact with the solution. 
(a)

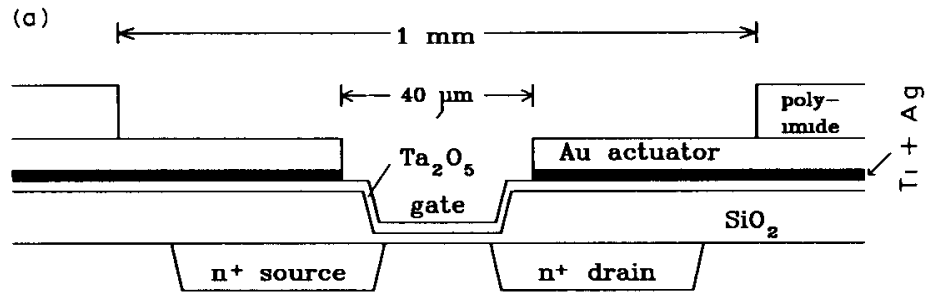

p substrate (b)

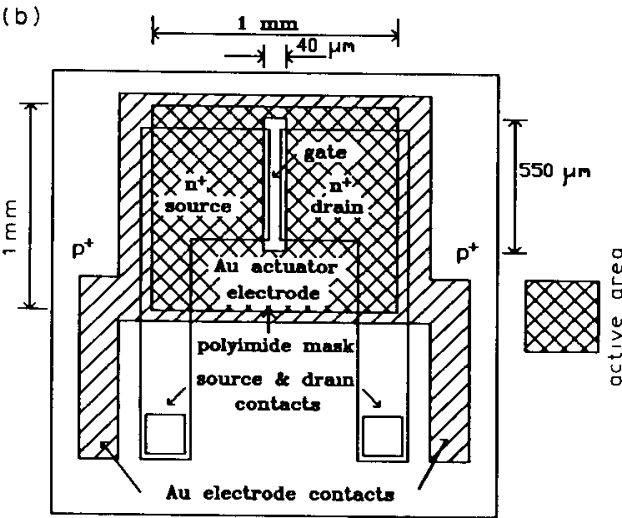

Fig. 7. (a) Cross-section and (b) top view of the sensor-actuator device with actuator electrode deposited around the gate.

The second type of actuator electrode consists of thin gold bridges over the ISFET gate area shaped as shown in Fig. 8ab. By photolithography and lift-off techniques, 27 gold bridges of $10 \mu \mathrm{m}$ width, spaced $10 \mu \mathrm{m}$ apart, are obtained. The distance between the gold actuator electrode bridges and the gate area is ca. $1 \mu \mathrm{m}$.

The chip $(3 \times 4 \mathrm{~mm})$ is glued on a piece of printed circuit board $(1 \times 10 \mathrm{~cm})$ together with a second ISFET chip. The bonding wires and the copper connection strips are protected by a synthetic resin. A thin gold film evaporated on a copper strip on the reverse side of the printed circuit board serves as a counter electrode for the supplied actuator current. In this way a dipsticklike structure of the device is obtained.

\section{Measurement set-up}

The measurement set-up is shown in Fig. 9. A second ISFET 1s used as a reference, which is possible because the bulk $\mathrm{pH}$ does not change during the experiment. The actuator current pulse is applied on one of the sensor-actuator devices only, and the gold "actuator" electrode shaped around the reference ISFET can now be used as a pseudo-reference electrode. A differential measurement of the ISFET output signals results in the desired signal, making an extra reference electrode superfluous.

All measurements were carried out in a $20-\mathrm{ml}$ vessel in a $0.1 \mathrm{M}$ potassium nitrate supporting electrolyte to avoid migration effects. After immersion of the dipstick sensor and addition of the (a)

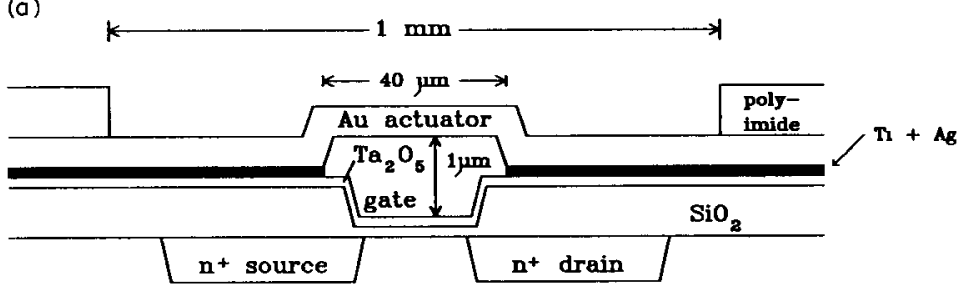

p substrate (b)

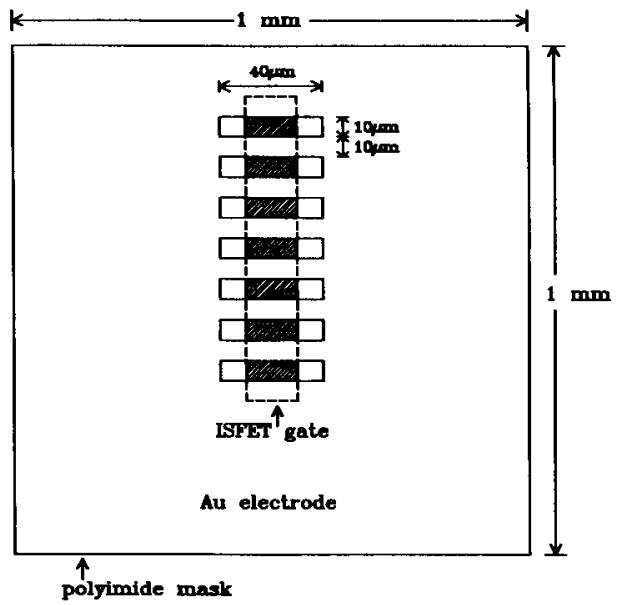

Fig. 8. (a) Cross-section and (b) top view of the actuator electrode of the sensor-actuator device with actuator electrode over the gate 


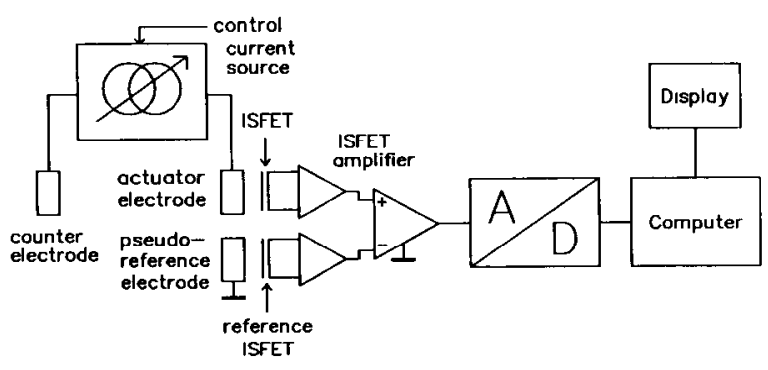

Fig. 9. Measurement set-up

acid, the concentration of which has to be determined, the solution is stirred. To avoid erroneous results due to convection, the measurement was started $1 \mathrm{~min}$ after the end of the stirring.

Following the actuator pulse, where the titrant is generated, a current pulse of opposite polarity is generated for two reasons: to homogenize the solution in the proximity of the actuator electrode by generating the same amount of $\mathrm{H}^{+}$ions as there are $\mathrm{OH}^{-}$ions generated during titration (or vice versa), and to avoid the formation of oxides on the gold actuator electrode after several anodic current pulses or to avoid the adsorption of hydrogen on the electrode surface after cathodic current pulses.

\section{RESULTS AND DISCUSSION}

During the generation of the titrant, the $\mathrm{pH}$-dependent output signal of the ISFET amplifier is sampled and the result is displayed on the computer CRT. A typical example for a coulometric titration is shown in Fig. 10.

The equivalence point $t_{\mathrm{eq}}$ is easily determined from the first derivative of the titration curve by calculating its minimum; $t_{\mathrm{eq}}$ is determined with an accuracy of $0.01 \mathrm{~s}$. The variation in a set of measurements is ca. $0.02 \mathrm{~s}$ for $t_{\mathrm{eq}}$ in the range $0.5-2.0 \mathrm{~s}$ up to $0.1 \mathrm{~s}$ for $t_{\mathrm{eq}}$ near the maximum of $t_{\text {eq }}\left(\right.$ ca. $10 \mathrm{~s}$ ). For larger values of $t_{\text {eq }}$ the variation increases as a result of convection the diffusion layer thickness for protons after $10 \mathrm{~s}$ of generation can be estimated from $6\left(D_{\mathrm{H}} t\right)^{1 / 2} \approx 1800 \mu \mathrm{m}$ [5]\}. This variation in $t_{\text {eq }}$ means that the bulk concentration can be determined with an accuracy of

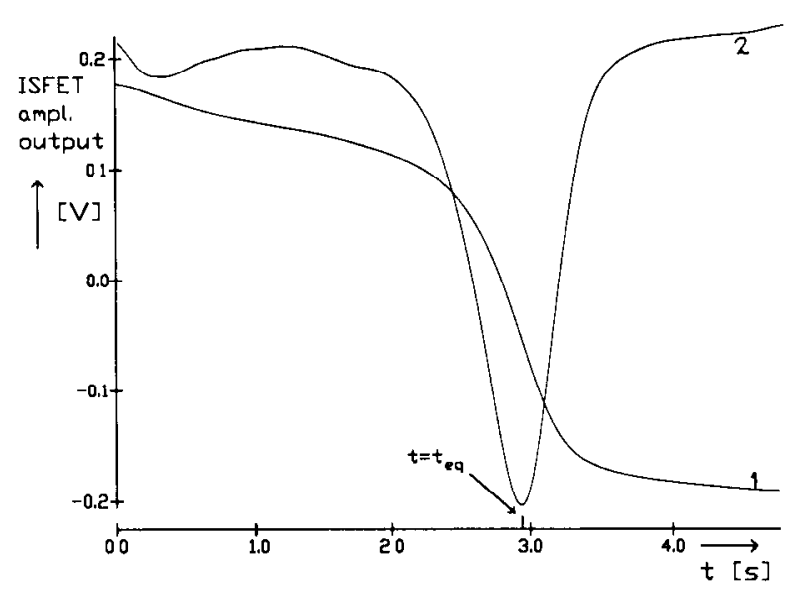

F1g. 10. Typical measured coulometric titration (curve 1) and its first derivative (curve 2)

ca. $40 \mu \mathrm{mol} 1^{-1}$ in the range $1-10 \mathrm{mmol} 1^{-1}$ using Eqn. 30, and the parameters given in Fig. 11.

A series of measurements with the sensor-actuator device as shown in Fig. 7 were done to determine $t_{\text {eq }}$ in solutions with different acetic acid concentration. The results are presented in Fig. 11, together with the calculated results of both the analytical model description, according to Eqn. 30 and the numerical solution, according to Eqns. 33, 34 and 38.

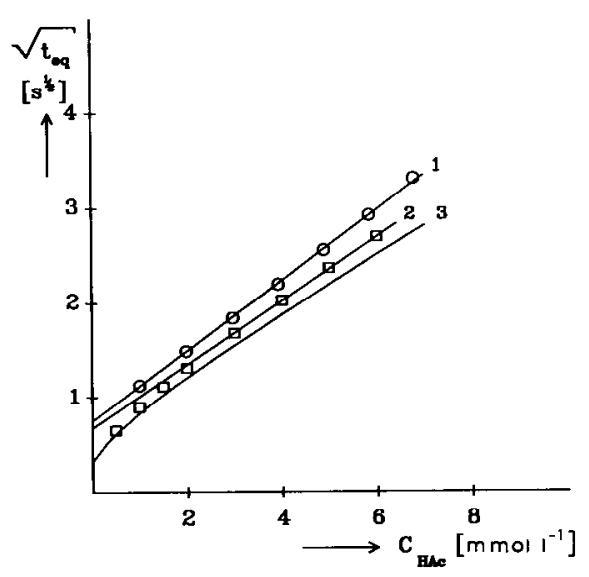

Fig. 11. Results of coulometric titration of HAc. Curve 1, measurement results; curve 2, results of numerical solution, curve 3, results of the analytical model. Actuator electrode shaped around the gate; $J_{\mathrm{c}}=10 \mathrm{~A} \mathrm{~m}^{-2} ; D_{\mathrm{HAc}}=1.21 \times 10^{-9}$, $D_{\mathrm{Ac}}=1.09 \times 10^{-9}, D_{\mathrm{H}}=9.33 \times 10^{-9}$ and $D_{\mathrm{OH}}=5.27 \times 10^{-9}$ $\mathrm{m}^{-2} \mathrm{~s}$. 
It is clear from Fig. 11 that the square root of the time needed to reach the equivalence point depends linearly on the acid concentration, apart form very low concentrations. Considering Eqn. 31 , this linear relationship can be expected.

The current dependence of the slope of the curve will be discussed separately; apart from the current, the slope depends on the diffusion coefficient of the titrated acid.

From the literature $[10,11]$, the diffusion coefficients for $\mathrm{HAc}, \mathrm{Ac}^{-}, \mathrm{OH}^{-}$and $\mathrm{H}^{+}$are obtained and used for both the numerical solution (curve 2) and the analytical model description (curve 3 ).

The slope of the curves representing the measurements and the numerical solution (curve 1 and curve 2 , respectively) are in good agreement. The slope of the third curve, representing the analytical model description, also fits the measurement results closely, but is systematically ca. $10 \%$ smaller than the measured curve. This is possibly caused by the fact that the establishment of equilibrium during diffusion of the partly dissociated acid is not taken into account in the analytical model description; a mean diffusion coefficient, composed of both the high $\mathrm{H}^{+}$diffusion coefficient and the relatively low HAc diffusion coefficient in a certain ratio, will always be higher than the HAc diffusion coefficient used in the analytical model description, resulting in an increased slope.

None of the three curves intersects the vertical axis of Fig. 11 at the origin in the absence of any acid in the bulk solution, owing to the non-zero distance between the actuator electrode and the sensor, which results in a delay time as described by Eqn. 29 .

By fitting the linearly extrapolated curves of the numerical solution and of the analytical model description for zero bulk concentration to the results of the measurements, the position of the sensor in the one-dimensional model description was estimated to be $x_{\mathrm{s}}=50 \mu \mathrm{m}$. The real distance between the sensor and actuator of the device is ca. $20 \mu \mathrm{m}$. Although direct comparison between this estimated distance and the real sensor-actuator distance of the device is not fully realistic (because a one-dimensional model description is used), the estimated $x_{\mathrm{s}}$ is of the same order of magnitude as the measured $x_{\mathrm{s}}$.

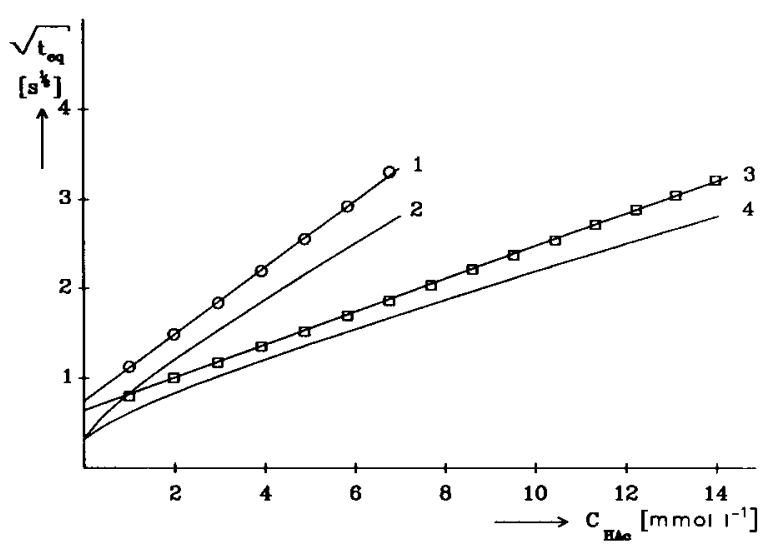

Fig 12 Results of coulometric titration of HAc. $J_{c}=10 \mathrm{~A}$ $\mathrm{m}^{-2}$ curve 1 , measurement results, curve 2 , results of the analytical model $J_{\mathrm{c}}=20 \mathrm{~A} \mathrm{~m}^{-2}$ : curve 3 , measurement results, curve 4, results of the analytical model Actuator electrode shaped around the gate, diffusion coefficients as in Fig 11

The analytical model description also predicts an inversely proportional relationship between the slope of the curve as given in Fig. 11 and the actuator current density $J_{\mathrm{c}}$.

Results for the same sensor-actuator device with $J_{c}$ increased by a factor 2 are presented in Fig. 12 and show a decrease of the slopes (in $\mathrm{s}^{1 / 2}$ $\mathrm{mol}^{-1}$ ) by a factor of $0.186 / 0.376=0.49$. This is in good agreement with the analytical model de-

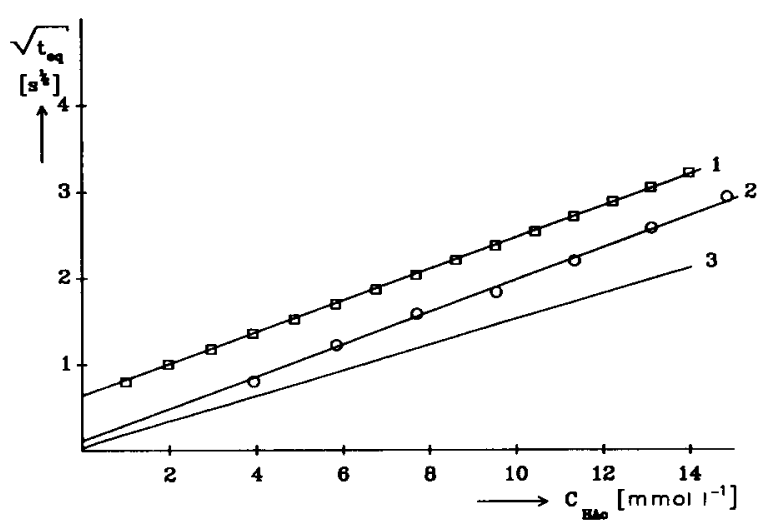

Fig. 13. Results of coulometric titration of HAc. Actuator electrode shaped around the gate, $J_{\mathrm{c}}=20 \mathrm{~A} \mathrm{~m}^{-2}$ : curve 1 , measurement results. Bridge-type actuator electrode, $J_{c}=20 \mathrm{~A}$ $\mathrm{m}^{-2}$. curve 2 , measurement results; curve 3 , results of the analytical model. Diffusion coefficients as in Fig. 11. 
scription, which predicts a decrease by a factor of 0.5 .

The experiments were repeated using a bridgetype actuator electrode as shown in Fig. 8. Because the distance between the sensor and the actuator electrode is now much smaller, a smaller delay time is expected (Fig. 13). The point of intersection of curve 2 with the vertical axis is indeed much smaller in comparison with the results of the device with the actuator electrode shaped around the gate (curve 1).

The result of the analytical model description is also given in curve 3 in Fig. 13. Curve 3 is fitted to curve 2 with respect to the linearly extrapolated delay time at the vertical axis intersect, resulting in the estimation of $x_{\mathrm{s}}=1.0 \mu \mathrm{m}$, which is in close agreement with the real sensor-actuator distance of ca. $1 \mu \mathrm{m}$.

The authors thank J.G. Bomer for expert technical assistance. B.H. v.d. S. thanks the Royal Netherlands Academy of Arts and Sciences for the award of a fellowship. This investigation is part of the research program of the Foundation for Fundamental Research on Matter (FOM), which is supported by the Netherlands Technology Foundation (STW).

\section{REFERENCES}

1 W Olthus, B.H. van der Schoot, F. Chavez and P. Bergveld, Sens Actuat, 17 (1989) 279.

2 L.J Bousse, P. Bergveld and W.E. van der Linden, in Proceedıngs, Scientıfic Session on Electrochemical Detecton in Flow Analysis, Matrafured, Hungary, October 1982, p 257.

3 B.H van der Schoot and P. Bergveld, Sens. Actuat., 13 (1988) 251.

4 B.H. van der Schoot and P. Bergveld, Anal. Chım Acta, 199 (1987) 157

5 A.J Bard and L.R. Faulkner, Electrochemıcal Methods, Fundamentals and Applications, Wiley, New York, 1980, pp. 253, 683 .

6 G. Astarita, Mass Transfer with Chemical Reaction, Elsevier, Amsterdam, 1967.

7 R B Bird, W.E Stewart and E.N Lightfoot, Transport Phenomena, Wiley, 1960, p 599

8 D. Britz, Digital Simulation in Electrochemistry, Lecture Notes in Chemistry 23, Springer, Berlın, 1981.

9 T. Matsuo and M. Esash,, Sens. Actuat., 1 (1981) 77

10 W.J. Moore, Physical Chemistry, Longman, London, 1972, p. 435.

11 V. Vitagliano and P A. Lyons, J. Am. Chem Soc., 78 (1956) 4538. 